\title{
Extracorporeal membrane oxygenation in adults
}

\section{JP Johnston}

Specialist Registrar in Anaesthesia, Regional Intensive Care Unit, Royal Victoria Hospital, Belfast, UK

TITLE Efficacy and economic assessment of conventional ventilatory support versus extracorporeal membrane oxygenation for severe adult respiratory failure (CESAR): a multicentre randomised controlled trial

AUTHORS Peek GJ, Mugford M,Tiruvoipati R et al.

JOURNAL Lancet 2009; 374:I35I-63. doi:I0.I0I6/S0 I40-6736(09)6I069-2

DECLARATION OF INTERESTS No conflict of interests declared.

\author{
Correspondence to JP Johnston, \\ Regional Intensive Care Unit, \\ Royal Victoria Hospital, \\ Grosvenor Road, \\ Belfast BTI2 6BA, UK \\ tel. +44 (0)28 90240503 \\ e-mail paulj_II@hotmail.com
}

\section{SUMMARY}

This paper is the summation of five years' work studying clinical and economic outcomes comparing the use of extracorporeal membrane oxygenation (ECMO) and conventional medical care to treat severe respiratory failure. Extracorporeal membrane oxygenation is an intervention, which, like cardiac bypass, can support a failing myocardium when used in a veno-arterial mode. This trial, Conventional ventilation or ECMO for Severe Adult Respiratory failure (CESAR), studied pulmonary support alone, using ECMO in the veno-venous mode.

Peek and colleagues screened 766 patients with respiratory failure between July 2001 and August 2006. A significant number were excluded, the most significant factors being ECMO bed availability, an inappropriate lung injury score (LIS) or difficult ventilation for greater than seven days. A total of 180 subjects were enrolled; 90 were randomised to the treatment limb and 90 to the conventional medical care limb. The primary outcomes studied were death or significant disability at six months post-randomisation. A holistic range of secondary outcome measures were also studied.

In an intention-to-treat analysis the primary outcome of death or severe disability (37\% vs $53 \%$ ) did reach statistical significance, despite some missing data relating to disability status in the conventional treatment limb. On further analysis mortality outcomes alone, without inclusion of disability scoring, at six months in the treatment vs conventional limbs ( $37 \%$ vs $45 \%, p=0.07)$, did not reach statistical significance.

Better outcomes were demonstrated in the treatment group despite only $76 \%$ of those randomised and transferred to a specialist centre actually receiving ECMO. The other $24 \%$, if they had not died prior to arrival at the treatment centre, received only conservative management strategies that would be available in most intensive care units. The study group therefore recommend the transfer of adult patients with acute severe respiratory failure to a centre with the ability to initiate an ECMO-based management protocol.

\section{OPINION}

Extracorporeal membrane oxygenation is not a new technique. Indeed, it has become accepted in neonatal medical practice as a standard of care. In the UK, an ECMO service is provided by six centres registered with the Extracorporeal Life Support Organisation (ELSO). Only one, Glenfield Hospital in Leicester, currently provides a service for adult patients. As such, the CESAR study was in essence a single-centre trial, despite individual control patients receiving care in their referring intensive care unit (ICU). Blinding was impossible as all ECMOrandomised patients were transferred to a single site. An inherent degree of bias cannot therefore be excluded.

Results from the CESAR trial demonstrate a trend towards improved survival using ECMO vs conventional care in adults with severe acute respiratory failure, defined by a Murray Lung Injury Score $>3.0$. This trend is not statistically significant, which is consistent with two major previous trials published in 1979 and 1994. ${ }^{1,2}$ In the CESAR trial, however, ECMO was shown to be beneficial when survival without severe disability was substituted as the primary endpoint. This significance could be greatly influenced by missing data classifying the disability status data for three patients in the conventional management group. These patients were simply lost to follow-up. However, as survival has not been shown to be significantly different between groups, it is hard to imagine why those who survive, post ECMO, do so with less severe disability.

When recruiting subjects, the authors used the LIS described by Murray et al. $^{3}$ rather than the definitions for acute lung injury (ALI) or acute respiratory distress syndrome (ARDS) produced following a 1994 consensus conference, ${ }^{4}$ a Murray score $>3$ being the primary inclusion factor. This is not a concern given previous evidence that these scoring systems can be used interchangeably. ${ }^{5}$ It is worth noting that such scores have not been shown in clinical trials to be predictive of mortality in severe respiratory failure. ${ }^{6}$ It is therefore reasonable to assume that a validated, mortalitypredictive scoring system, if it existed, could better 
stratify for inclusion in a study with mortality as one of the primary outcomes.

Other compounding factors are evident when reviewing the paper. Attention should be paid to statistically different rates of steroid use, low-volume ventilation and diuresis to dry weight between groups. These interventions were performed to a greater extent in the treatment limb and might be considered beneficial at various stages of lung injury. In particular, the beneficial effect of a low-volume ventilatory strategy has been well demonstrated to improve outcomes in ARDS.? The detrimental effect of a relatively high-volume ventilation strategy in the conventional treatment group may even be significant enough to explain the relative benefit demonstrated in the treatment group. Another factor which may have had a significant effect was the use of a molecular albumin recirculating system in the treatment limb. As a mode of therapy used to bridge patients until liver transplant, it would not appear to be of specific benefit in the setting of severe respiratory failure, but this does add another significant unknown factor, being used in $20 \%$ of patients in the ECMO limb.

A potential detrimental effect is noted in the treatment limb related to the pre-ECMO protocol allowing transfusion to a packed cell volume of $40 \%$. Transfusion of critically ill patients to a haemoglobin concentration of approximately $13.3 \mathrm{~g} / \mathrm{dl}$ would not be standard practice. Following the TRICC study ${ }^{8}$ and more recent meta-analyses on the subject describing the detrimental effects of over-transfusion, the protocol-driven liberal transfusion policy may have influenced the CESAR trial's survival results.

The cost of treatment with ECMO is estimated at more than twice the cost of conventional treatment. Given that the risk of death is not statistically different, it is difficult to justify the routine use of this form of therapy. Those who survive ECMO seem to do so with less disability and this could have an economic benefit in terms of qualityadjusted life years. However, as an explanation for the lower degree of disability remains outstanding, this economic argument cannot yet be used to justify more widespread use of this treatment modality.

\section{REFERENCES}

I Zapol WM, Snider MT, Hill JD et al. Extracorporeal membrane oxygenation in severe acute respiratory failure. A randomized prospective study. JAMA 1979; 242:2193-6. doi:I0.100I/ jama.242.20.2193

2 Morris AH, Wallace CJ, Menlove RL et al. Randomized clinical trial of pressure-controlled inverse ratio ventilation and extracorporeal $\mathrm{CO}_{2}$ removal for adult respiratory distress syndrome. Am J Respir Crit Care Med 1994; 149:295-305.

3 Murray JF, Matthay MA, Luce JM et al. An expanded definition of the adult respiratory distress syndrome. Am Rev Respir Dis 1988; 138:720-3; erratum in: Am Rev Respir Dis 1989; I39:1065.

4 Bernard GR, Artigas A, Brigham KL et al. The American-European Consensus Conference on ARDS. Definitions, mechanisms, relevant outcomes, and clinical trial coordination. Am J Respir Crit Care Med 1994 Mar; 149:8I8-24.
The CESAR trial does provide some positive results. Reported survival rates of $63 \%$ in the ECMO limb and $51 \%$ in the conventional limb are improved compared with historical mortality figures for acute severe respiratory failure in similar studies. Mortality rates in the 1970s for patients with acute severe respiratory failure were typically in the order of $90 \%$. What has also been successfully demonstrated is the relative safety of the entire ECMO process. Significant levels of iatrogenic harm have not been highlighted, in contrast to the levels of morbidity reported in previous trials. ${ }^{1,2}$

As more patients survive, critical illness consideration of the need for post-ICU care is pertinent. The authors have meticulously gathered data documenting various health outcomes as secondary outcome measures. The results add evidence to the need for continued care following hospital discharge. The healthcare outcomes point toward clinically significant levels of respiratory dysfunction, disability, anxiety, depression and chronic pain. Many surviving patients will therefore consume significant healthcare resources and require input from a wide range of healthcare professionals to facilitate rehabilitation.

When conducting a randomised control trial of life-saving therapy, in a heterogeneous group, there will be potential shortcomings. This is not a new phenomenon in the setting of ICU trials and in this case the shortcomings are largely insurmountable. Considering the group of patients and the intervention under scrutiny, the trial has been well designed and executed. It has tried to answer two very difficult questions: is ECMO in severe adult respiratory failure both worthwhile and cost-effective? I believe that it has not provided enough evidence to justify the conclusion drawn from the study. However, as a treatment of last resort, particularly during the recent HINI epidemic, it has likely been invaluable and has without doubt saved lives. This alone justifies the existence and potentially the expansion of an ECMO service. This study provides good phase 2 data from a single centre, but needs a large multicentre trial to confirm benefit before ECMO can be recommended as routine treatment. At present it should remain a salvage therapy.

5 Meade MO, Guyatt GH, Cook RJ et al. Agreement between alternative classifications of acute respiratory distress syndrome. Am J Respir Crit Care Med 200I; 163:490-3.

6 K Atabai, M A Matthay. The pulmonary physician in critical care: Acute lung injury and the acute respiratory distress syndrome: definitions and epidemiology. Thorax 2002; 57:452-8. doi:I0.II36| thorax.57.5.452

7 The Acute Respiratory Distress Syndrome Network.Ventilation with lower tidal volumes as compared with traditional tidal volumes for acute lung injury and the acute respiratory distress syndrome. $N \mathrm{Engl}$ J Med 2000;342:I30I-8. doi:I0.I056/NEJM20000504342 I80 I

8 Hébert PC. Transfusion requirements in critical care (TRICC): a multicentre, randomized, controlled clinical study. Transfusion Requirements in Critical Care Investigators and the Canadian Critical Care Trials Group. Br J Anaesth 1998; 8I (Suppl I):25-33. 